\title{
A utilização das redes sociais como ferramenta na monitoria acadêmica: relato de experiência
}

The use of social networks as a tool in academic monitoring: experience report

El uso de las redes sociales como herramienta en el seguimiento académico: relato de experiencia

Recebido: 26/01/2021 | Revisado: 27/01/2021 | Aceito: 11/02/2021 | Publicado: 20/02/2021

Julielen Larissa Alexandrino Moraes ORCID: https://orcid.org/0000-0003-3753-6645 Universidade Federal do Pará, Brasil E-mail: julielen.moraes@gmail.com

Pâmela Moraes Ferreira

ORCID: https://orcid.org/0000-0002-4094-2014 Universidade Federal do Pará, Brasil

E-mail: pammoraferr@gmail.com

Aline Maria Pereira Cruz Ramos

ORCID: https://orcid.org/0000-0001-8812-2923 Universidade Federal do Pará, Brasil E-mail: nurse.alinecruz@gmail.com

Cintia Yolette Urbano Pauxis Aben Athar ORCID: https://orcid.org/0000-0002-6951-3547 Universidade Federal do Pará, Brasil E-mail: abenathar_cintia@hotmail.com

Fabianne de Jesus Dias de Sousa ORCID: https://orcid.org/0000-0002-8151-3507 Universidade Federal do Pará, Brasil E-mail: fabianneSousa@hotmail.com

\begin{abstract}
Resumo
A utilização das redes sociais intensificou-se em tempos de Pandemia da COVID-19 e, tornou-se uma ferramenta digital que contribui para a construção e disseminação do conhecimento científico. $\mathrm{O}$ estudo teve como objetivo relatar as experiências de monitoria acadêmica na atividade curricular Atenção Integral a Saúde do Adulto e Idoso. Trata-se de um estudo descritivo, do tipo relato de experiência, realizado a partir da vivência de discentes na monitoria de uma atividade curricular em ensino remoto emergencial. A experiência de monitoria permitiu aos discentes conhecer o processo de ensino-aprendizagem, inclusive a utilização e o aumento da utilização das redes sociais. Dessa maneira, foi criado uma página no Instagram denominada@idosopaid'egua com informações acerca da promoção do envelhecimento ativo e saudável possibilitando a troca de informações entre a comunidade acadêmica e idosos, além de favorecer a educação em saúde da população geral acerca do processo de envelhecimento. Além do que, potencializou-se a construção de aprendizados científicos e interpessoais de forma bidirecional, entre os acadêmicos e participantes da rede social, enfatizando a importância da enfermagem na saúde do idoso visando a promoção da saúde no processo de envelhecimento. Constatou-se a utilização das redes sociais promove uma maior sensibilização da população acerca do processo de envelhecimento, além de uma adequada capacitação dos monitores envolvidos, incentivando ações que visem à promoção da saúde da população idosa.
\end{abstract}

Palavras-chave: Uso de redes sociais; Ensino superior; Envelhecimento saudável; Educação em enfermagem.

\begin{abstract}
The use of social networks has intensified in the pandemic times of COVID-19 and has become a digital tool that contributes to the construction and dissemination of scientific knowledge. The study aimed to report the experiences of academic monitoring in the curricular activity Comprehensive Attention to Adult and Elderly Health. This is a descriptive study, of the experience report type, carried out from the experience of students in the monitoring of a curricular activity in remote emergency teaching. The monitoring experience allowed students to learn about the teaching-learning process, including the use and increased use of social networks. In this way, a page on Instagram was created called@idosopaid'égua with information about the promotion of active and healthy aging, enabling the exchange of information between the academic community and the elderly, in addition to promoting health education for the general population about the aging. In addition, the construction of scientific and interpersonal learning in a bidirectional way was enhanced, among academics and participants in the social network, emphasizing the importance of nursing in the health of the elderly aiming at promoting health in the aging process. It was found that the use of
\end{abstract}


social networks promotes a greater awareness of the population about the aging process, in addition to an adequate training of the monitors involved, encouraging actions aimed at promoting the health of the elderly population.

Keywords: Use of social networks; Higher education; Healthy aging; Nursing education.

\section{Resumen}

El uso de las redes sociales se ha intensificado en los tiempos de la pandemia del COVID-19 y se ha convertido en una herramienta digital que contribuye a la construcción y difusión del conocimiento científico. El estudio tuvo como objetivo reportar las experiencias de seguimiento académico en la actividad curricular de Atención Integral a la Salud de Adultos y Ancianos. Se trata de un estudio descriptivo, del tipo relato de experiencia, realizado a partir de la experiencia de los estudiantes en el seguimiento de una actividad curricular en la enseñanza a distancia de emergencia. La experiencia de seguimiento permitió a los estudiantes conocer el proceso de enseñanza-aprendizaje, incluido el uso y mayor uso de las redes sociales. De esta manera, se creó una página en Instagram denominada @idosopaid'égua con información sobre la promoción del envejecimiento activo y saludable, posibilitando el intercambio de información entre la comunidad académica y las personas mayores, además de promover la educación en salud para la población en general sobre el proceso de envejecimiento. Además, se potenció la construcción de aprendizajes científicos e interpersonales de forma bidireccional, entre académicos y participantes de la red social, destacando la importancia de la enfermería en la salud de las personas mayores con el objetivo de promover la salud en el proceso de envejecimiento. Se encontró que el uso de las redes sociales promueve una mayor conciencia de la población sobre el proceso de envejecimiento, además de una adecuada formación de los monitores involucrados, impulsando acciones encaminadas a promover la salud de la población mayor.

Palabras clave: Uso de redes sociales; Educación superior; Envejecimiento saludable; Educación en enfermería.

\section{Introdução}

No Brasil em 2020, a população idosa é estimada em 30 milhões de habitantes. Entre os estados da região Norte, destaca-se o Pará, com 793.740 habitantes idosos (IBGE,2016). Na capital, Belém, ocorre a maior concentração, com aproximadamente 257 mil idosos (17,1\% da população) caracterizando o aumento da longevidade nessa região (IBGE,2020).

A Organização Mundial da Saúde (OMS) define envelhecimento saudável como o "processo de desenvolvimento e manutenção da capacidade funcional que permite o bem-estar na idade avançada” (WHO,2015).

A doença do Novocoronavírus 2019 conhecida também como COVID-19 surgiu inicialmente em Wuhan, província de Hubei, na China, em dezembro de 2019 e, evoluiu rapidamente, mundialmente, para pandemia. Impactando negativamente para a população geral com graves efeitos em todas as áreas inclusive na educacional (Malik et al., 2020).

A população mundial utiliza o isolamento social para achatar a curva de transmissão e evitar o colapso dos sistemas de saúde. Devido a rápida transmissibilidade, a taxa de internação hospitalar poderia aumentar exponencialmente e causar a saturação dos serviços de saúde de tal modo que seria inviável atender todos (Ferrari \& Cunha, 2020).

O distanciamento social fez com que as universidades brasileiras interrompessem suas atividades de ensino, pesquisa e extensão. Dessa forma, tornaram- se necessárias estratégias para a manutenção das atividades de ensino e de assistência à saúde, destacando-se a substituição das atividades presenciais de ensino e atendimento à saúde pelo uso das Tecnologias da Informação e Comunicação (TICs) (Lopes \& Heimann, 2016).

As TICs têm sido utilizadas com frequência no cotidiano das pessoas, sobretudo e intencionalmente na educação. Assim, quando são utilizadas com o objetivo de tornar os espaços de construção de conhecimento diferenciados e diversificar os processos e metodologias de aprendizagem, podem proporcionar uma aproximação da universidade com a realidade do cotidiano do graduando (Bertusso, 2020).

A utilização das redes sociais intensificou-se nesse período pandêmico por se tratar de plataformas com alta velocidade na geração de dados com postagens feitas em todos os momentos (Xavier et al.,2020).

A Universidade Federal do Pará (UFPA) por meio da por meio da Pró-Reitoria de Ensino e Graduação (PROGRAD), estimula as atividades de monitoria, atrelado ao tripé do ensino, visando fortalecer a relação entre corpo docente e discente, além de proporcionar ao acadêmico a oportunidade de se preparar para atividades de ensino. 
A monitoria é definida como uma ferramenta de aprimoramento acadêmico, em que proporciona ao aluno-monitor, sua ampliação nos conhecimentos, integração entre teoria e prática, revisão dos conteúdos, esclarecimento de dúvidas, e, ainda, permite a aproximação ao exercício da docência (Andrade et al.,2018).

Dessa maneira, a monitoria permite a utilização de metodologias ativas definidas como estratégias educativas que proporcionam ao graduando a construção do conhecimento científico por meio de um processo de ensino-aprendizagem crítico e reflexivo, estimulando a autonomia e participação produtiva no aprendizado (Brito et al.,2017).

Assim, temas relacionados à saúde do idoso como o processo de envelhecimento humano, políticas públicas à pessoa idosa e, promoção do envelhecimento ativo e saudável, são temas relevantes, densos tornando o processo ensino-aprendizagem exaustivo.

Nesse sentido, a introdução de metodologias ativas proporciona aos graduandos aprendizado dinâmico, objetivo e criativo. Em tempos de pandemia de COVID-19, essas metodologias tornam-se uma ferramenta imprescindível no auxílio do aprendizado no ensino superior.

Assim, objetivou-se relatar a experiência de discentes de uma monitoria acadêmica em saúde do idoso em ações para promoção do envelhecimento saudável desenvolvida por meio de metodologia ativa (redes sociais) no curso de graduação em enfermagem tendo em vista o ensino remoto emergencial (ERE).

\section{Metodologia}

Trata-se de um estudo com abordagem qualitativa, descritiva, do tipo relato de experiência. Refere-se como abordagem qualitativa métodos utilizados em que a interpretação feita por pesquisadores sobre determinados fenômenos estudados é importante para a comunidade de uma forma geral (Pereira et al.,2018).

As atividades de monitoria acadêmica foram realizadas na atividade curricular de atenção integral à saúde do adulto e idoso (AISAI), vinculada ao curso de Enfermagem da UFPA como atividade de ensino pela PROGRAD, em Belém, PA, Brasil, no período entre os meses agosto e dezembro do correspondendo ao semestre letivo de 2020.2, por duas alunasmonitoras e a professora-coordenadora.

Essa atividade curricular Atenção Integral a Saúde do Adulto e Idoso possui carga horaria total de 119hs divididas em aulas teóricas (34hs) e aulas prática (85hs), sendo ofertada no terceiro semestre do curso, onde são abordadas nas aulas teóricas, as temáticas envolvendo políticas públicas, processo de envelhecimento humano, condições clínicas, instrumentos de avaliação e família e cuidadores. Quanto as aulas práticas são desenvolvidas ações na atenção primaria a saúde e ambulatório de geriatria em hospital de alta complexidade.

Inicialmente foi elaborado um edital para a seleção de bolsistas para a monitoria acadêmica com as seguintes fases: estar regularmente matriculado na instituição, ter cursado a atividade curricular, análise do currículo comprovado na área da atividade curricular, carta de intenção e coeficiente de rendimento na atividade curricular Atenção Integral a saúde do Adulto e Idoso com rendimento igual ou superior a 7,0 com disponibilidade de 20horas/semanais para o desenvolvimento das atividades de monitoria.

No decorrer do ano de 2020, vivenciamos a pandemia de COVID-19, assim, as aulas presenciais foram substituídas pelo ensino remoto emergencial (ERE) (Portaria $n^{\circ} 544,2020$ ) e, as práticas foram suspensas enquanto durar a pandemia, consequentemente, a comunidade acadêmica deparou-se com o ensino virtual, plataformas digitais e aplicação de metodologias ativas. Assim, os encontros de monitoria passaram a ser virtuais e, a utilização das redes sociais tornou-se uma ferramenta de ensino-aprendizagem.

Após o término da monitoria acadêmica, foi realizada uma avaliação das atividades desenvolvidas entre as monitoras e a professora-coordenadora, assim, a rede social @idosopaid'égua continuará “ativa” no Instagram com o intuito de continuar 
dando contribuições sobre o processo de envelhecimento humano. Finalmente, o processo avaliativo deu-se através da participação dos graduandos nas atividades bem como nas interações ocorridas na rede social (Instagram) por meio da @idosopaid'égua.

\section{Resultados e Discussão}

A experiência da monitoria acadêmica constituiu-se como uma iniciação à docência, em que as alunas-monitoras passaram a conhecer, sob supervisão, as atividades inerentes à atividade docente. Inicialmente, fez-se um cronograma das atividades remotas pela professora coordenadora, no qual foi sugerido a utilização das metodologias ativas, tendo como estratégia o uso das redes sociais afim de facilitar o processo de assimilação e socialização de conhecimento.

Nesse sentido o uso de tecnologias digitais, como as redes sociais, incentiva o desenvolvimento de novas competências digitais, como a autonomia, a automotivação, a reflexão crítica, a capacidade de análise e a tomada de decisões diante de situações-problema, o trabalho em equipe, o uso de diferentes linguagens e o fazer colaborativo (Haag et al., 2008). Assim, a monitoria acadêmica realizou suas atividades, com as devidas adaptações em razão do isolamento social ocasionado pela pandemia de COVID-19, entretanto, seguindo as recomendações do ERE.

$\mathrm{O}$ uso de plataformas digitais contribui no aumento da interação entre professores e discentes, por meio da pesquisa e discussão, a fim de que possam ser construídos individualmente e coletivamente seus conhecimentos (Varella, Vermelho \& Hesketh, 2002). Dessa forma as monitoras juntamente com a professora coordenadora elaboraram uma página na rede social Instagram intitulada @idosopaid'égua tendo como finalidade a divulgação de assuntos abordados na atividade curricular, de linguagem técnica, porém acessível a comunidade geral. Nessa atividade pode-se observar a interação entre os discentes, de forma colaborativa nas atividades e, não apenas a fragmentação de tarefas.

As atividades foram divididas entre as monitoras na busca de materiais científicos visando postagens diárias abordando assuntos semanais inclusos no cronograma da atividade curricular. A interação se dava por meio da rede social com boa aceitação dos graduandos de diversas áreas da saúde, sugestões de filmes, livros e poesias eram exemplos dessas interações. Piaget (2002), cita a importância das atividades em grupos, as pesquisas, o estímulo à autonomia do aluno, expondo que as relações necessitam se alicerçar em respeito mútuo, reciprocidade e cooperação.

Foram abordadas pelas monitoras, diversas temáticas ao longo do semestre acadêmico relacionados a atividade curricular saúde do adulto e idoso do curso de graduação em enfermagem. Os assuntos abordados foram divididos em temáticas como a intergeracionalidade, o suporte social ao idoso, a sexualidade na terceira idade, gerontecnologia, a importância da promoção do envelhecimento ativo e saudável, como transformar uma cidade amiga do idoso, saúde mental na pessoa idosa, a situação do idoso institucionalizado, abordagem de doenças neurológicas como por exemplo, Alzheimer, o câncer de próstata, o papel do cuidador de idoso e família, as síndrome geriátricas, o aumento da polimedicação entre idosos, o mercado de trabalho na terceira idade, a necessidade de exercícios físicos para prevenção e tratamento de doenças, e o ageísmo na contemporaneidade, como a discriminação contra os idosos ainda é extremamente presente e deplorável.

A educação em saúde realizada por meio da rede social Instagram, tem se mostrado eficiente na medida em que são apresentadas novas temáticas em relação à saúde do idoso para os seguidores (Machado et al.,2020).

Foram ainda, publicadas postagens pelas monitoras, acerca de datas comemorativas alusivas a pessoa idosa com o intuito de dar maior visibilidade e conscientização dos assuntos de interesse para a população idosa, como por exemplo o dia internacional do idoso, dia mundial da saúde mental, dia do professor, dia mundial do combate a diabetes, dia da consciência negra e do dia mundial da luta contra AIDS.

As redes sociais associadas ao ensino superior contribuem para a relevância na formação entre os participantes proporcionando a construção de conhecimentos científicos e novas habilidades. Além disso, o ensino à saúde pode desenvolver 
estratégias tecnológicas-educativas, nas quais possam intervir no processo de ensino-aprendizagem, permitindo a abordagem de temas antes restritos à comunidade acadêmica, entretanto, com o aumento do uso das ferramentas digitais devido a pandemia COVID-19 esses assuntos passam a ser maiores difundidos e compartilhados a todos de um modo geral contribuindo para a difusão do conhecimento, nesse caso da monitoria, de temas relacionados à pessoa idosa.

\section{Considerações Finais}

$\mathrm{O}$ uso das redes sociais no auxílio de atividades de ensino, como a monitoria acadêmica mostrou-se relevante para o desenvolvimento de ações em saúde bem como a construção de conhecimento da comunidade acadêmica e da população idosa acerca das alterações sobre o processo de envelhecimento humano.

Desse modo a associação dessa ferramenta digital com a monitoria permitiu permuta de informação para a população desde aspectos conceituais, como sua própria avaliação, até o incentivo para a promoção de um envelhecimento ativo e saudável. Além do que com o surgimento da pandemia causada pelo novo coronavírus (SARS-CoV-2) houve uma mudança no processo de ensino-aprendizagem, com implantação do ensino remoto emergencial no ensino superior.

Diante do exposto, o presente estudo proporcionou as monitoras, acadêmicos da enfermagem aprendizados e reflexões da importância da monitoria acadêmica no compromisso científico à comunidade de forma integral, além de perceberem o processo de envelhecimento, necessita de atividades que valorizem os conhecimentos, visando à promoção da saúde da pessoa idosa.

\section{Agradecimentos}

À Universidade Federal do Pará por meio da Pró-Reitoria de Ensino e Graduação (PROGRAD) pelas concessões das bolsas de monitoria (Edital 001/2020).

\section{Referências}

Andrade, E. G. R., Rodrigues, I. L. A., Nogueira, L. M. V., \& Souza, D.F. (2018). Contribuição da monitoria acadêmica para o processo ensino aprendizagem na graduação em enfermagem. Rev Bras Enferm, 71 (Supl4),1596-603.

Bertusso, F. R., Machado, E. de G., Terhaag, M. M. \& Malacarne, V. (2020). A utilização das Tecnologias de Informação e Comunicação (TICs) no ensino de Ciências: um paradigma a ser vencido. Research, Society and Development, 9(12), e26691211099.

Portaria № 544 de junho de 2020. Dispõe sobre a regulação do ensino remoto emergencial. Diário Oficial da União,114,1, 62.

Brito, L. S., Ribeiro, L. S., Ulisses, L. O., Ortiz, M. F. A., \& Whitaker, C. O. M. (2017). Experiência de discentes de enfermagem em metodologias ativas na atividade de ensino docente. Rev Baiana Enferm, 31,1-8.

Ferrari, A. \& Cunha, A. M. (2020). A pandemia de Covid-19 e o isolamento social: saúde versus economia. Andrés Ferrari.

Haag, G. S., Kolling, V., Silva, E., Melo, S., \& Pinheiro, M. (2008). Contribuições da monitoria no processo ensino-aprendizagem em enfermagem. Rev Bras Enferm, 61(2), 215-220.

Instituto Brasileiro de Geografia e Estatística (2020). Sistema IBGE de Recuperação Automática - SIDRA. População por grupo de idade, município, $1^{\circ}$ trimestre de 2020. https://sidra.ibge.gov.br/tabela/5918\#resultado.

Instituto Brasileiro de Geografia e Estatística (2016). Síntese de indicadores sociais uma análise das condições de vida da população brasileira. Rio de Janeiro: IBGE.

Lopes, J. E. \& Heimann, C. (2016). Uso das tecnologias da informação e comunicação nas ações médicas a distância: um caminho promissor a ser investido na saúde pública. Journal of Health Informatics, 8(1), 26-30. http://www.jhi-sbis.saude.ws/ojs-jhi/index.php/jhi-sbis/article/view/364/252

Machado, A. L. L. B., Misquita, M. S., Alexandre, Érica R., Silva, F. M. R. da, Rodrigues, C. de S., Andrade, M. G., Silva, P. G. da, Silva, D. M. H. de S., Alves, M. T., Sousa, A. B. de A. G., Braz, G. de A., Crisóstomo, L.S., Sousa, F.A.B. de ., Camelo, L.O., Santos, T. E. M., Araújo, G. M., Morais, M. A. M., Norte, H. B. F., Pinto, A. L. \& Melo , D. F. C. (2020). Utilização das redes de comunicação social para a promoção e empoderamento da saúde da mulher: relato de experiência. Research, Society and Development, 9(11), e77291110548.

Malik, Y. S., Sircar, S., Bhat, S., Sharun, K., Dhama, K., Dadar, M., Tiwari, R. \& Chaicumpa, W. (2020). Emerging novel coronavirus (2019-nCoV)-current scenario, evolutionary perspective based on genome analysis and recent developments. Veterinary Quaterly, 40(1), 68-76. 
Research, Society and Development, v. 10, n. 2, e38710212360, 2021

(CC BY 4.0) | ISSN 2525-3409 | DOI: http://dx.doi.org/10.33448/rsd-v10i2.12360

Varella, P. G., Vermelho, S. C., Hesketh, C. G. (2002). Aprendizagem Colaborativa em ambientes virtuais de aprendizagem: a experiência inédita da PUC-PR. Revista Diálogo Educacional, 3(6),11-27.

Pereira, A. S., Shitsuka, D. M., Parreira, F. J. \& Shitsuka, R. (2018). Metodologia da pesquisa científica. UFSM. https://repositorio.ufsm.br/bitstream/ha ndle/1/15824/Lic_Computacao_Metodologia-Pesquisa-Cientifica.pdf?sequence=1.

Piaget, J. (2002). Para onde vai a educação? Rio de Janeiro: José Olympio.

Xavier, F., Olenscki, J. R. W., ACosta, A. L., Sallum, M. A. M., \& Saraiva, A. M. (2020). Análise de redes sociais como estratégia de apoio à vigilância em saúde durante a Covid-19. Estudos Avançados, 34(99), 261-282.

World Health Organization (2015). World report on ageing and health. Geneva: WHO. http://apps.who.int/iris/bitstream /10665/186463/1/9789240694811_eng. pdf?ua=1 